\title{
The correlation between unexplained infertility and exosomes
}

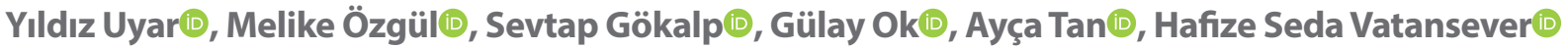 \\ Manisa Celal Bayar University Medical Faculty, Manisa, Turkey
}

\begin{abstract}
Objectives: Endometrial receptivity plays the most important role for successful implantation. Increasing endometrial receptivity may improve infertility and increase Assisted Reproductive Technologies success. The aim of this study was to investigate the effect of exosome specific markers CD63 and CD9 which are promising molecules in the pathogenesis and treatment of many diseases on endometrial receptivity in women with unexplained infertility.

Material and methods: This prospective study was conducted between November 2015 and March 2017. Proliferation and secretion periods of endometrial samples from fertile and infertile cases were collected. The paraffin-embedded tissue sections were stained with hematoxylin-eosin for the immunohistochemical analysis distributions of CD63 and CD9.

Results: The results of this study demonstrated that the CD63 immunoreactivity was higher in both luminal and glandular epithelium of infertile patients when compared with fertile patients during the proliferative phase $(p=0.009, p=0.008)$. In the infertile proliferation phase, endometrium CD9 immunoreactivity was rarely detected in both the luminal and glandular epithelium. In the secretion phase of endometrium, CD9 immunoreactivity was mild in fertile patients, the increased immunoreactivity of $C D 9$ was observed in both luminal and glandular epithelium of infertile patients $(p=0.037, p=0.037)$.

Conclusions: Increased levels of CD63 in infertile proliferation phase endometrium should represent an unfavorable signaling. Moreover, the increased levels of CD9 in infertile secretion phase endometrium could be used as a biomarker to evaluate endometrial receptivity. These exosome-specific markers can be considered as potential molecular markers of infertility.
\end{abstract}

Key words: exosomes, endometrium, fertility, infertility, embryo implantation

\section{INTRODUCTION}

Infertility is a condition that has psychological, economic and medical effects which cause trauma and stress especially on the people who desire to have children [1]. Infertility is a reproductive system disease defined by the inability to achieve clinical pregnancy after unprotected sexual intercourse for 12 months or more $[2,3]$. Unexplained infertility is the absence of identifiable causes for infertility [3].

Endometrial receptivity plays a critical role for successful implantation, and impaired endometrial receptivity may lead to subfertility and limit the success of Assisted Reproductive Technologies (ART) [4]. Implantation, a critical step in the establishment of pregnancy, ensues under the uterine fluid microcirculation signals which contain various proteins, lipids and other molecules secreted from the endometrium and possibly from the fallopian tubes and blastocyst (such as hCG) [5]. Unexplained infertility has been suggested to be caused by a disorder in molecular and cellular biomarkers in endometrial receptivity [6]. In studies on unexplained infertility, which is explained as a high failure of implantation after ART, pro-inflammatory factors and interleukins have been shown in uterine wall invasion [7].

Extracellular vesicles contain exosomes, microvesicles and apoptotic bodies and they are also a heterogeneous group of particles defined by their size, composition and density. The smallest extracellular vesicles are exosomes (30-100 nm) released by plasma membrane fusion of multivesicular bodies containing intraluminal vesicles $[8,9]$.

Exosomes which are nanoparticles capable of specifically transferring small RNAs and messenger RNA (mRNAs) through the extracellular medium to cells located in distant regions can be isolated from culture supernatants of cell 
lines or from various body fluids [10]. According to cells, the exosomal content may vary. However, different cell-based exosomes also express certain common exosome specific proteins. Among these proteins are CD9, CD37, CD53, CD63, CD81 and CD82 tetraspanin molecules [10]. Exosomes can be characterized and purified via their specific cell surface markers like tetraspanins CD63 and CD9 which are responsible for exosome formation. Moreover, exosomes present signal transduction (EGFR), antigen presentation (MHCI and $\mathrm{MHC}$ II) and other transmembrane proteins (LAMP1) on their surface $[10,11]$. Exosome secretion has been demonstrated in a number of cell types, including embryonic stem cells and in vitro produced embryos [12]. Some studies have shown that exosomes in the uterine cavity or slightly larger microvesicles (100-300 $\mathrm{nm}$ ) are released from the endometrial epithelium. These exosomes or microvesicles contain specific miRNAs so that these miRNAs can be transferred to the trophectoderm cells or endometrial epithelial cells to promote implantation $[13,14]$.

\section{Objectives}

The aim of this study was to investigate the distribution of exosomes in endometrial samples taken during proliferation and secretion periods from fertile and unexplained infertile patients and their effects on implantation in unexplained infertility.

\section{MATERIAL AND METHODS}

\section{Study Design and Experimental Groups}

The study was conducted using fertile ( $n=5$ proliferation phase and $n=5$ secretion phase) and infertile ( $n=5$ proliferation phase and $n=5$ secretion phase) patients who were between 25- and 38-years age. Endometrial specimens were obtained by probe curettage or by pipelling during proliferation and secretion period of the menstrual cycle from both fertile and infertile patients. Menstrual cycle phase was confirmed by histological dating [15]. Prior to commencing with study, ethical permission was taken from Health Science Ethics Committee and written informed consent was obtained from all participants.

\section{Selection of Patient Groups}

The current study involved female participants aged between 25-38 years. Participants were selected from amongst patients that were admitted to our hospital Obstetrics and Gynecology Clinic.

Participants in the fertile group had at least one child, did not have any uterine disease, had dysfunctional uterine bleeding, and had to undergo curettage during a non-hemorrhagic period. The infertile group consisted of participants who had no children, had been married for at least 1 year and were not able to achieve clinical pregnancy after unprotected sexual intercourse for 12 months. Infertile cases were determined based on clinical diagnosis, anamnesis, radiological and ultrasound assessments.

Either endometrial evaluation for diagnosis or treatment, curettage materials from infertile and fertile patients were taken under anesthesia via probe curettage or pipelined endometrial sampling. Samples were collected from patients that were clinically diagnosed as fertile or infertile with dysfunctional uterine bleeding.

In the current study, the menstrual cycle-related intake of the samples is essential.Thus, any other organic endometrial changes outside the dysfunctional uterine bleeding were deactivated and standard sample uptake was achieved. Patients with a pathologic diagnosis of an organic cause such as hyperplasia, neoplasia or polyps, or an organic lesion such as myoma in the examination and who had been using steroid hormones for at least six months prior to the study were not included in this study.

As a result of endometrial sampling, patients with endometrium in the proliferation phase and secretion phase were chosen among the patients who admitted to our gynecology outpatient clinic with the complaint of abnormal bleeding and diagnosed with dysfunctional uterine bleeding excluded from organic reasons and whose BHCG negative on day of endometrial sampling included in our study.

The current study consists of two groups of patients, fertile and infertile. The endometrial samples taken from both patient groups were divided further into fertile proliferation (Group 1), infertile proliferation (Group 2), fertile secretion (Group 3) and infertile secretion (Group 4) groups.

\section{Histological Evaluation}

The endometrium tissue samples were fixed in $10 \%$ neutral formalin and then embedded in paraffin using standard protocols. After processing, samples were embedded in paraffin and $5 \mu \mathrm{m}$ sections were taken. Sections were used for both histochemical and immunohistochemical analyses.

\section{Histochemical Analyses}

For morphological evaluation, sections taken from the paraffin blocks at $5 \mu \mathrm{m}$ thickness with a rotary microtome were deparaffinized overnight at $60^{\circ} \mathrm{C}$ (Nuve, FN 400), followed by chemical deparaffinization with xylene for 1 hour. The sections were passed through the decreasing alcohol series (95\%, 80\%, 70\% and 60\% alcohol series) for 2 minutes each and the sections were treated for 6 minutes with Hematoxylin (Leica, 3801562E) solution. Sections were washed for 5 minutes under water and then stained with Eosin (Bio-Optica, 380610) for 1.5 minutes after 
differentiating with $1 \%$ acid-alcohol solution. Sections were taken up into xylene by passing through increasing alcohol series ( $80 \%$ and $95 \%)$. The sections which were left in xylene for 1 hour were covered with cover glasses (Marienfeld, 01 01060) using entellan solution (Merck, UN 1866) and examined under a light microscope (Olympus BX43).

\section{Immunohistochemical Analyses}

For immunohistochemical evaluation of the CD63 and CD9, avidin-biotin-peroxidase indirect immunohistochemistry method was used. After deparaffinization of sections as above, they were washed with phosphate buffered saline (PBS) and $3 \% \mathrm{H}_{2} \mathrm{O}_{2}(\mathrm{H} 1009$, Sigma-Aldrich, USA) was applied. They were then washed with PBS again. For antigen retrieval, they were incubated with trypsin for 10 minutes in $37^{\circ} \mathrm{C}$ and then washed with PBS. Sections were then incubated with blocking serum for $1 \mathrm{~h}$ and, anti-CD63 (sc-5275, lot \#D0115, Santa Cruz, USA), and anti-CD9 (sc-13118, lot \#K1814, Santa Cruz, USA) primer antibodies in a 1/50 dilution for both antibodies were added and incubated at $4^{\circ} \mathrm{C}$ for overnight. After the washing step with PBS, the secondary antibodies biotin (30 $\mathrm{min}$ ) and streptavidin (30 min) were applied respectively (Histostain ${ }^{\oplus}$-Plus Bulk Kit Cat No: 85-9043- Invitrogen, Carlsbad, California, United States). Immunoreactivity was visualized by the application of $50 \mu \mathrm{l}$ DAB chromogen (Histostain-Plus IHC Kit, DAB, broad spectrum Cat No. 859643, Invitrogen, Carlsbad, California, United States). After washing with distilled water, they were counter stained with Mayer's hematoxylin and covered with immunohistochemistry mounting medium. Immunohistochemical process was repeated 3 times. Immunohistochemical staining was scored using a semi-quantitative analysis is based on the calculation of HSCORE $=\sum \mathrm{Pi}(i+1)(\mathrm{i}=$ intensity of staining and $\mathrm{Pi}$ is the percentage of positively stained cells for each intensity) formula after evaluation of intensities as 1 (mild), 2 (moderate) or 3 (strong) [16-19]. For each antibody, five different fields were evaluated by at least two investigators independently under light microscope (BX43, Olympus), blinded to the source of the samples as well as to each other's results and the average score was then utilized. Results were given as median after analyzing statistically with Mann-Whitney $U$ test for differences among groups. When the $p$ value was $<0.05$, the data was considered significant.

\section{RESULTS \\ Histological Results}

After hematoxylin and eosin staining, endometrial luminal and glandular epithelium were observed in all groups. The luminal epithelium was a single-layered columnar epithelium during proliferation phases of both the fertile and infertile patients. The glands in the connective

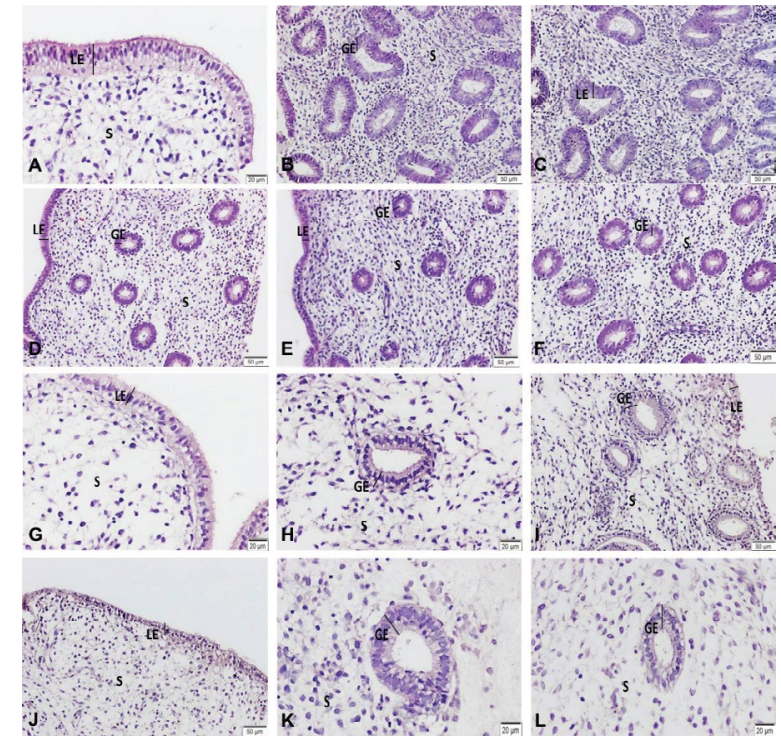

Figure 1. Hematoxylin and eosin staining of endometrium. Proliferative phase of fertile patients $(A-C)$, proliferative phase of infertile patients $(D-F)$, secretion phase of fertile patients $(G-l)$, secretion phase of infertile patients (J-L). (A, G, H, K, L Scale Bars: $20 \mu \mathrm{m})(B, C, D, E, F$, I and J Scale Bars: $50 \mu \mathrm{m})$

tissue of the lamina propria were round in shape and had single-layered columnar epithelium in both fertile and infertile patients in proliferative phase (Fig. 1A-F). In the secretory phase of the fertile and infertile patients' endometrium, the luminal epithelium was a single-layered columnar epithelium and the gland structures appeared as glycogen vacuoles in the basal parts of the glandular columnar epithelium (Fig. 1G-L).

\section{Immunohistochemical Results}

In the proliferative phase of the fertile patients' endometrium, weak immunoreactivity of CD63 was detected in both the luminal (HSCORE = 190) (min-max; 170-200) and glandular epithelium (HSCORE = 170) $($ min-max; 150 205), and also mild CD63 immunoreactivity was detected in the stroma (Fig. 2A, B). In the proliferative phase of the infertile patients' endometrium, increased (mild/strong) CD63 immunoreactivity was detected in both the luminal (HSCORE = 310) (min-max; 300-325) and glandular epithelium (HSCORE = 315) (min-max; 310-320) when compared to the fertile group, they were statistically significant ( $p=0.009, p=0.008$ ) (Tab. 1 and Fig. 3). Intensity of CD63 immunoreactivity in stroma was seen similar in both fertile and infertile group (Fig. 2C, D). In the secretion phase of the fertile patient's endometrium, weak and mild immunoreactivity of CD63 was detected in the luminal (HSCORE $=175)(\min -\max ; 165-175)$ and glandular epithelium (HSCORE = 285) (min-max; 255-305) respectively, mild CD63 immunoreactivity was also observed in the stroma (Fig. 2E, F). In the secretion phase of the 


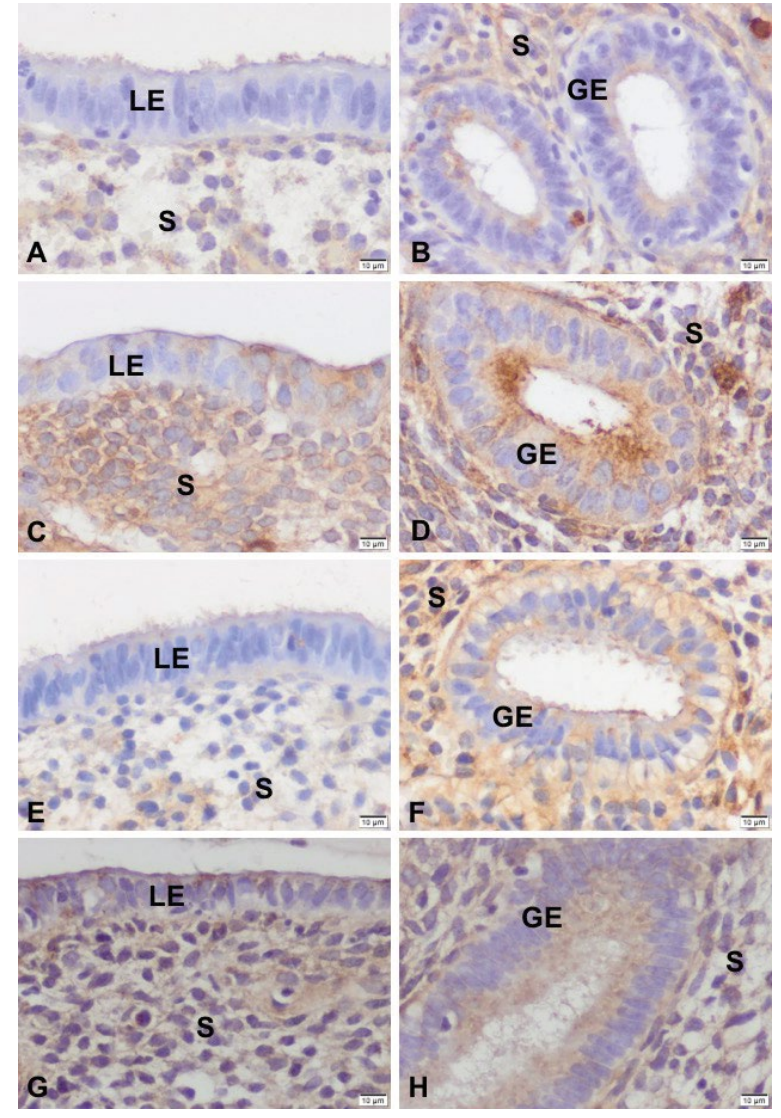

Figure 2. Immunohistochemical distribution of CD63. Scale Bars: $10 \mu \mathrm{m}$

infertile patient's endometrium, increased (mild/moderate) CD63 immunoreactivity was detected in both luminal $($ HSCORE $=200)($ min-max; 200-200) and glandular epithelium $($ HSCORE = 250) (min-max; 250-250) when compared with fertile group, however, this intensity was slightly less than the proliferative phase of the infertile endometrium (Fig. 2G, H) and when compare the results it was statistically significant ( $p=0.034, p=0.037$ ) (Tab. 1 and Fig. 3 ). In addition, intensity of CD63 in the stroma of the infertile secretion phase of the endometrium (mild) decreased when compared with other groups (Fig. 2).

CD9 immunoreactivity was observed as very weak in the infertile proliferation phase of endometrial luminal $(H S C O R E=130)(\min -\max ; 115-135)$ and glandular epithelium $(H S C O R E=140)($ min-max; 100-160) (Fig. 4C, D). This immunoreactivity was weaker then fertile proliferation luminal epithelium (HSCORE = 150) $(\min -\max ; 130-170)(p=0.025)$. In contrast to that, $C D 9$ immunoreactivity was not observed in the fertile proliferation glandular epithelium (Fig. 4A, B). It was evident that there were significant differences between fertile and infertile proliferation gland epithelium $(p=0.005)$ (Tab. 1 and Fig. 5). In stroma, CD9 immunoreactivity was weak in fertile proliferation endometrium, but not observed in infertile proliferation tissue samples. In the infertile secre-

\begin{tabular}{|c|c|}
\hline \multicolumn{2}{|l|}{ CD63 } \\
\hline Compared Groups & p Value \\
\hline $\begin{array}{l}\text { Fertile proliferation vs infertile proliferation (luminal } \\
\text { epithelium) }\end{array}$ & $p=0.009$ \\
\hline $\begin{array}{l}\text { Fertile proliferation vs infertile proliferation (glandular } \\
\text { epithelium) }\end{array}$ & $p=0.008$ \\
\hline $\begin{array}{l}\text { Fertile secretion vs infertile secretion } \\
\text { (luminal epithelium) }\end{array}$ & $p=0.034$ \\
\hline $\begin{array}{l}\text { Fertile secretion vs infertile secretion } \\
\text { (glandular epithelium) }\end{array}$ & $p=0.037$ \\
\hline \multicolumn{2}{|l|}{ CD9 } \\
\hline Compared Groups & p Value \\
\hline $\begin{array}{l}\text { Fertile proliferation vs infertile proliferation (luminal } \\
\text { epithelium) }\end{array}$ & $p=0.025$ \\
\hline $\begin{array}{l}\text { Fertile proliferation vs infertile proliferation (glandular } \\
\text { epithelium) }\end{array}$ & $p=0.005$ \\
\hline $\begin{array}{l}\text { Fertile secretion vs infertile secretion } \\
\text { (luminal epithelium) }\end{array}$ & $p=0.037$ \\
\hline $\begin{array}{l}\text { Fertile secretion vs infertile secretion } \\
\text { (glandular epithelium) }\end{array}$ & $p=0.037$ \\
\hline
\end{tabular}

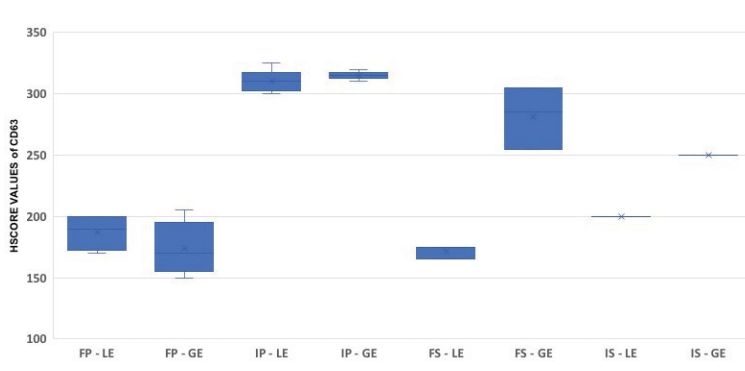

Figure 3. HSCORE values of CD63 immunoreactivities. FP — Fertile Proliferation; IP — Infertile Proliferation; FS - Fertile Secretion; IS - Infertile Secretion; LE — Luminal Epithelium; GE - Glandular Epithelium

tion phase, CD9 immunoreactivity was weak/moderate in the luminal $(H S C O R E=220)(\min -\max ; 220-220)$ and the glandular epithelium $($ HSCORE = 220) $($ min-max; 220-220) (Fig. 4G, H), whereas CD9 immunoreactivity of fertile secretion luminal $($ HSCORE $=150)(\min -\max ; 130-170)$ and glandular epithelium $($ HSCORE = 160) (min-max; 160-170) was weak (Fig. 4E, F). It was clearly observed that there were significant differences between fertile and infertile secretion phase epitheliums ( $p=0.037, p=0.037$ ) (Tab. 1 and Fig. 5). In stroma, CD9 immunoreactivity was very weak in the fertile secretion tissues, but not observed in infertile secretion tissues.

\section{DISCUSSION}

In our study, we showed that there were differences in the release of CD63 and CD9 membrane surface proteins 


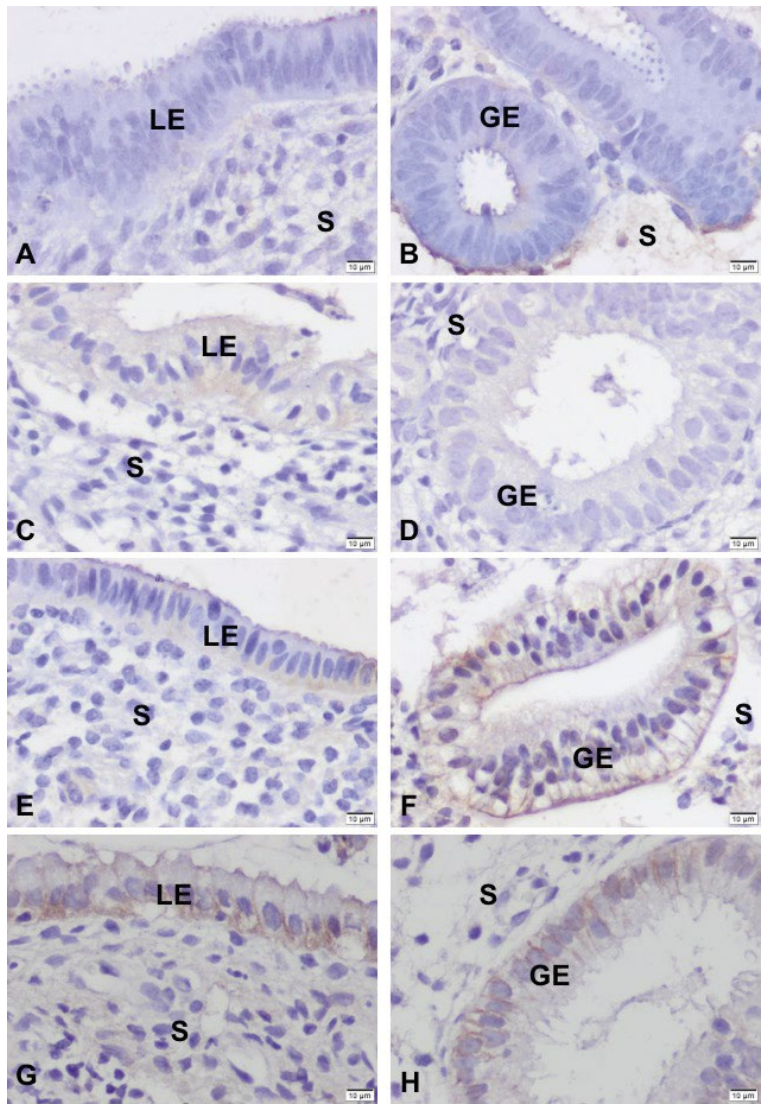

Figure 4. Immunohistochemical distribution of CD9. Scale Bars: $10 \mu \mathrm{m}$

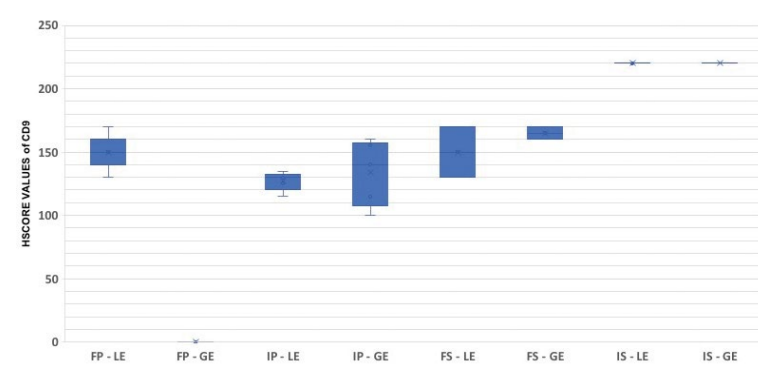

Figure 5. HSCORE values of CD9 immunoreactivities. FP — Fertile Proliferation; IP — Infertile Proliferation; FS - Fertile Secretion; IS — Infertile Secretion; LE — Luminal Epithelium; GE — Glandular Epithelium

used to characterize exosomes in luminal and glandular epithelium of the endometrium between the unexplained infertility and the fertile women.

To date, many researchers have been stated that it is well known the endometrial epithelium is the first maternal surface to interact with the implanting embryo. And they also stated that the inclusion of extracellular vesicles in the implantation process is a relatively new phenomenon [14, 20-24]. In many studies; extracellular vesicles containing specific RNAs, including microRNAs and proteins, have been released into the uterine cavity and transferred to tropho- blast cells or endometrial epithelial cells where they promote implantation [13, 14, 21, 25]. Ng YH et al. [14] showed for the first time that the tetraspanins, CD9 and CD63 used as cell surface markers of exosomes that exist on the surface of fertile endometrial epithelial cells and they contain specific miRNAs in the endometrial epithelium and stated that the exosomes and/or exosome-derived miRNA could be used as biomarkers for endometrial receptivity.

Our results demonstrated that CD63 expression in the secretion phase endometrium of fertile group was higher in the glandular epithelium compared to the luminal epithelium, and this intensity was statistically significant. It was observed that intensity of CD63 in luminal and glandular epithelial in the infertile proliferation phase group was higher when compared to the fertile proliferation phase group, and also this increased immunoreactivity was statistically significant. However, significantly decreased CD63 expression was detected during infertile secretion phase compared to the infertile proliferation phase.

$\mathrm{Ng} \mathrm{YH}$ et al. [14]; in their study, showed that CD63 and CD9 had strong apical staining in the luminal and glandular epithelial cells of fertile women, and interestingly, this staining for CD63 reached the highest levels in the mid-secretory period, which is the time of endometrial receptivity and they said it might be important in implantation. Therefore, they stated that enough secretion of increased exosome production in the mid-secretory period was needed for the implantation window and if this process could not proceed properly, result with implantation failure. As a result, $\mathrm{Ng} \mathrm{YH}$ et al. [14]; have expressed that exosomes modulate the behavior of the immune system and cancer cells and because the embryo implantation is in common with the behavior of immune system and cancer cells, the clarification of the function of the exosomes in the uterine cavity will extend our understanding of the endometrial-embryo cross talk and infertility.

In our study, we found that CD63 expression was similar and weak in the glandular and luminal epithelium of the fertile proliferative phase endometrium's, whereas CD63 expression in the glandular epithelium in the fertile secretion phase increased significantly. It is thought that during implantation, CD63 has an especially increased in glandular epithelium, and the luminal secretion of CD63 is similar in the proliferation and secretion phases of fertile group, so that CD63 secreted from the glandular epithelium may have a controlling role in implantation.

According to the results of our study, CD63 could not be accepted as an indicator or biomarker of endometrial signaling pathways in the infertile group due to the CD63 expression levels in the infertile group during the proliferation phase, furthermore the CD63 secretion was slightly increased in luminal epithelium but decreased 
in glandular epithelium in the infertile secretion phase compared to the fertile secretion phase. However, the observed increase of proliferation phase CD63 in the infertile group compared to the fertile group suggests that exosomal trafficking increases in the endometrial epithelium and it may cause negative effects in order to control different signal pathways before implantation.

Burnett LA et al. [26] stated in their review that several studies have demonstrated that interactions between the embryo and the uterine microenvironment are important for successful implantation and healthy pregnancy $[12,14$, 27, 28]. In their studies, Rosenbluth EM et al. [12], have shown that some exosomal miRNAs can be secreted by human embryos in IVF cycles and that these exosome-derived miRNAs can be secreted into the IVF culture environment that can be used as a biomarker in predicting IVF success and outcome of pregnancy.

Our study showed that CD9 immunoreactivity in the glandular epithelium in the fertile proliferation phase was significantly different compared to the luminal epithelium. Thus, CD9 expression was thought to be secreted mainly in the luminal epithelium of fertile proliferative phase. However, in the luminal epithelium of infertile proliferative phase, the CD9 immunoreactivity decreased and it was statistically significant compared to the fertile group. In the infertile proliferative phase, CD9 immunoreactivity was significantly expressed in the glandular epithelium.

It was clearly seen that the CD9 expression in the glandular epithelium of fertile secretion group increased compared to the fertile proliferation phase significantly, however the increase of CD9 immunoreactivity in the infertile secretion phase compared to both fertile groups and infertile proliferation phase group is higher. CD9 immunoreactivity was increased in the infertile secretion phase compared to the fertile secretion phase and this increase was statistically significant. The distribution of CD9 in both luminal and glandular epithelium of infertile secretion phase was also found to be higher than the infertile proliferation phase and it was statistically significant.

Expression of CD9 in the fertile secretion phase supports the presence of exosomes for endometrial regulation before implantation in both luminal and glandular epithelium. In contrast, in the infertile secretion phase, the CD9 increase in both luminal and glandular epithelium significantly increased, suggesting that the exosomal traffics were greater in both epithelium of infertile endometrium may be cause of unexplained infertility.

Iwai et al. [29] showed that changes in CD9 localization due to cellular activity in mice and human uterine secretions and stated that these changes may be related to infertility. Chaudhari-Kank MS et al. [30] showed that expression of CD9 had decreased in endometrial stromal cells of infertile women. They also stated that this decrease in CD9 expression could cause to infertility by impairing implantation. In the study of Chaudhari-Kank MS et al. [30], all cases were only in the secretory period. In our study, proliferation and secretion periods of infertile cases were evaluated and CD9 in stroma, on this subject in both phases expression was observed similar to the results of Chaudhari-Kank MS et al. study which is one of the most recent studies.

According to our study's results, we haven't thought that CD63 could be used as a biomarker in infertility. However, due to the significant increase of CD9 in the secretory phase of infertile group, it may be possible to use CD9 to assess infertility related issues or as a biomarker in terms of endometrium.

\section{CONCLUSIONS}

Based on the findings of this study increased CD9 reactivity in infertile secretion phase endometrium could be used as a biomarker which could be an unfavorable signaling of infertility or increased expression for recovery. In addition the increase of CD63 in the infertile proliferation group suggested that exosomal expression in the endometrial epithelium before implantation was changed and may have negative effects.

It can be stated that endometrial differences of exosomes may affect implantation and the results of our study may shed light on future studies. To predict the use of exosomes in infertility, particularly CD9 as a biomarker, further studies are needed to be done.

\section{Conflict of interest}

The authors declare that they have no conflict of interests.

\section{REFERENCES}

1. Kumar N, Singh AK. Trends of male factor infertility, an important cause of infertility: A review of literature. J Hum Reprod Sci. 2015; 8(4): 191-196, doi: 10.4103/0974-1208.170370, indexed in Pubmed: 26752853.

2. Zegers-Hochschild F, Adamson GD, de Mouzon J, et al. International Committee for Monitoring Assisted Reproductive Technology, World Health Organization, International Committee for Monitoring Assisted Reproductive Technology, World Health Organization. The International Committee for Monitoring Assisted Reproductive Technology (ICMART) and the World Health Organization (WHO) Revised Glossary on ART Terminology, 2009. Hum Reprod. 2009; 24(11): 2683-2687, doi: 10.1093/humrep/dep343, indexed in Pubmed: 19801627.

3. Zegers-Hochschild F, Adamson GD, Dyer S, et al. The International Glossary on Infertility and Fertility Care, 2017. Fertil Steril. 2017; 108(3): 393-406, doi: 10.1016/j.fertnstert.2017.06.005, indexed in Pubmed: 28760517.

4. Cakmak H, Taylor HS, Cakmak H, et al. Molecular mechanisms of treatment resistance in endometriosis: the role of progesterone-hox gene interactions. Semin Reprod Med. 2010; 28(1):69-74, doi: 10.1055/s0029-1242996, indexed in Pubmed: 20104430.

5. Salamonsen LA, Edgell T, Rombauts LJF, et al. Proteomics of the human endometrium and uterine fluid: a pathway to biomarker discovery. Fertil Steril. 2013; 99(4): 1086-1092, doi: 10.1016/j. fertnstert.2012.09.013, indexed in Pubmed: 23043689.

6. Sharkey AM, Smith SK. The endometrium as a cause of implantation failure. Best Pract Res Clin Obstet Gynaecol. 2003; 17(2): 289-307, doi: 10.1016/s1521-6934(02)00130-x, indexed in Pubmed: 12758101. 
7. Ozkan ZS, Deveci $D$, Kumbak $B$, et al. What is the impact of Th1/Th2 ratio, SOCS3, IL17, and IL35 levels in unexplained infertility? J Reprod Immunol. 2014; 103: 53-58, doi: 10.1016/j.jri.2013.11.002, indexed in Pubmed: 24368037.

8. Dear JW, Street JM, Bailey MA. Urinary exosomes: a reservoir for biomarker discovery and potential mediators of intrarenal signalling. Proteomics. 2013; 13(10-11): 1572-1580, doi: 10.1002/pmic.201200285, indexed in Pubmed: 23129434.

9. Stahl PD, Raposo G. Extracellular Vesicles: Exosomes and Microvesicles, Integrators of Homeostasis. Physiology (Bethesda). 2019; 34(3): 169-177, doi: 10.1152/physiol.00045.2018, indexed in Pubmed: 30968753.

10. Raposo G, Stoorvogel W. Extracellular vesicles: exosomes, microvesicles, and friends.J Cell Biol.2013; 200(4):373-383, doi: 10.1083/jcb.201211138, indexed in Pubmed: 23420871.

11. Abels ER, Breakefield XO. Introduction to Extracellular Vesicles: Biogenesis, RNA Cargo Selection, Content, Release, and Uptake. Cell Mol Neurobiol. 2016; 36(3): 301-312, doi: 10.1007/s10571-016-0366-z, indexed in Pubmed: 27053351.

12. Rosenbluth EM, Shelton DN, Wells LM, et al. Human embryos secrete microRNAs into culture media--a potential biomarker for implantation. Fertil Steril. 2014; 101(5): 1493-1500, doi: 10.1016/j. fertnstert.2014.01.058, indexed in Pubmed: 24786747.

13. Tannetta D, Dragovic R, Alyahyaei $Z$, et al. Extracellular vesicles and reproduction-promotion of successful pregnancy. Cell Mol Immunol. 2014; 11(6): 548-563, doi: 10.1038/cmi.2014.42, indexed in Pubmed: 24954226.

14. $\mathrm{Ng} \mathrm{YH}$, Rome $\mathrm{S}$, Jalabert $\mathrm{A}$, et al. Endometrial exosomes/microvesicles in the uterine microenvironment: a new paradigm for embryoendometrial cross talk at implantation. PLoS One. 2013; 8(3): e58502, doi: 10.1371/journal.pone.0058502, indexed in Pubmed: 23516492.

15. Noyes RW, Hertig AT, Rock J, et al. Dating the endometrial biopsy. Am J Obstet Gynecol. 1975; 122(2): 262-263, doi: 10.1016/s00029378(16)33500-1, indexed in Pubmed: 1155504.

16. Thike AA, Chng MJ, Fook-Chong $S$, et al. Immunohistochemical expression of hormone receptors in invasive breast carcinoma: correlation of results of $\mathrm{H}$-score with pathological parameters. Pathology. 2001; 33(1): 21-25, indexed in Pubmed: 11280603.

17. Ishibashi H, Suzuki T, Suzuki S, et al. Sex steroid hormone receptors in human thymoma. J Clin Endocrinol Metab. 2003; 88(5): 2309-2317, doi: 10.1210/jc.2002-021353, indexed in Pubmed: 12727990.

18. Hirsch FR, Varella-Garcia M, Bunn PA, et al. Epidermal growth factor receptor in non-small-cell lung carcinomas: correlation between gene copy number and protein expression and impact on prognosis. J Clin Oncol. 2003; 21(20): 3798-3807, doi: 10.1200/JCO.2003.11.069, indexed in Pubmed: 12953099.
19. John T, Liu G, Tsao MS. Overview of molecular testing in non-smallcell lung cancer: mutational analysis, gene copy number, protein expression and other biomarkers of EGFR for the prediction of response to tyrosine kinase inhibitors. Oncogene. 2009; 28 Suppl 1: S14-S23, doi: 10.1038/onc.2009.197, indexed in Pubmed: 19680292.

20. Altmäe $S$, Koel M, Võsa U, et al. Meta-signature of human endometrial receptivity: a meta-analysis and validation study of transcriptomic biomarkers. Sci Rep. 2017; 7(1): 10077, doi: 10.1038/s41598-017-10098-3, indexed in Pubmed: 28855728.

21. Vilella F, Moreno-Moya JM, Balaguer N, et al. Hsa-miR-30d, secreted by the human endometrium, is taken up by the pre-implantation embryo and might modify its transcriptome. Development. 2015; 142(18): 3210-3221, doi: 10.1242/dev.124289, indexed in Pubmed: 26395145.

22. Machtinger R, Laurent LC, Baccarelli AA. Extracellular vesicles: roles in gamete maturation, fertilization and embryo implantation. Hum Reprod Update. 2016; 22(2): 182-193, doi: 10.1093/humupd/dmv055, indexed in Pubmed: 26663221

23. Saadeldin IM, Oh HJu, Lee BC. Embryonic-maternal cross-talk via exosomes: potential implications. Stem Cells Cloning. 2015; 8: 103-107, doi: 10.2147/SCCAA.S84991, indexed in Pubmed: 26185458.

24. Evans J, Salamonsen LA, Winship A, et al. Fertile ground: human endometrial programming and lessons in health and disease. Nat Rev Endocrinol. 2016; 12(11): 654-667, doi: 10.1038/nrendo.2016.116, indexed in Pubmed: 27448058.

25. Burns $G$, Brooks $K$, Wildung $M$, et al. Extracellular vesicles in luminal fluid of the ovine uterus. PLoS One. 2014; 9(3): e90913, doi: 10.1371/journal. pone.0090913, indexed in Pubmed: 24614226.

26. Burnett LA, Nowak RA. Exosomes mediate embryo and materna interactions at implantation and during pregnancy. Front Biosci (Schol Ed). 2016; 8: 79-96, doi: 10.2741/s448, indexed in Pubmed: 26709898.

27. Kropp J, Salih SM, Khatib H. Expression of microRNAs in bovine and human pre-implantation embryo culture media. Front Genet. 2014; 5: 91, doi: 10.3389/fgene.2014.00091, indexed in Pubmed: 24795753.

28. Baig S, Lim JY, Fernandis AZ, et al. Lipidomic analysis of human placental syncytiotrophoblast microvesicles in adverse pregnancy outcomes. Placenta. 2013; 34(5): 436-442, doi: 10.1016/j. placenta.2013.02.004, indexed in Pubmed: 23465879.

29. Iwai M, Hamatani T, Nakamura A, et al. Membrane protein CD9 is repositioned and released to enhance uterine function. Lab Invest. 2019; 99(2): 200-209, doi: 10.1038/s41374-018-0145-1, indexed in Pubmed: 30401958

30. Chaudhari-Kank M, Zaveri K, Antia V, et al. Comparison of CD9 \& CD146 markers in endometrial stromal cells of fertile \& infertile females. Indian Journal of Medical Research. 2018; 147(6): 552, doi: 10.4103/ijmr.ijmr_1186_16. 